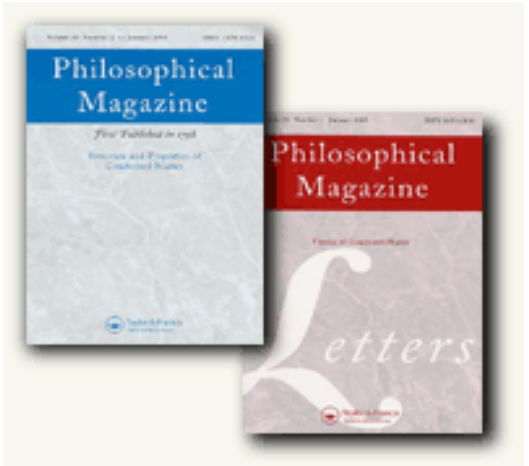

\title{
Cell-constrained melt-quench simulation of d-AICoNi: Ni-rich versus Co-rich structures
}

\begin{tabular}{|c|c|}
\hline Journal: & Philosophical Magazine \& Philosophical Magazine Letters \\
\hline Manuscript ID: & TPHM-10-Jun-0327 \\
\hline Journal Selection: & Philosophical Magazine \\
\hline $\begin{array}{r}\text { Date Submitted by the } \\
\text { Author: }\end{array}$ & $30-J u n-2010$ \\
\hline Complete List of Authors: & $\begin{array}{l}\text { Mihalkovic, Marek; Slovak Academy of Sciences, Institute of Physics } \\
\text { Henley, Christopher; Cornell University, Physics } \\
\text { Widom, M.; Carnegie Mellon University }\end{array}$ \\
\hline Keywords: & $\begin{array}{l}\text { modelling, molecular dynamic simulations, Monte-Carlo, } \\
\text { quasicrystalline alloys }\end{array}$ \\
\hline Keywords (user supplied): & \\
\hline
\end{tabular}

\section{S ScholaronE \\ Manuscript Central}


Philosophical Magazine Letters

Vol. 00, No. 00, Month 20xx, 1-9

\title{
RESEARCH ARTICLE
}

\section{Cell-constrained melt-quench simulation of d-AlCoNi: Ni-rich versus Co-rich structures}

\author{
M. Mihalkovič ${ }^{\text {a }}$ M. Widom ${ }^{\mathrm{b}}$, and C. L. Henley ${ }^{\mathrm{c}}$, \\ ${ }^{a}$ Inst. of Physics, Slovak Academy of Sciences, 84511 Bratislava, Slovakia \\ ${ }^{\mathrm{b}}$ Dept. of Physics, Carnegie Mellon Univ., Pittsburgh PA 15213, USA \\ c Dept. of Physics, Cornell Univ., Clark Hall, Ithaca, NY 14853-2501 USA
}

(30 June 2010)

\begin{abstract}
Complex crystalline ground state structures may be obtained by direct (simulated) quenches from the melt for systems of up to a few hundred atoms, given the constraint of a fixed unit cell, coupled with use of (i) replica-exchange Monte Carlo and (ii) realistic empirical interaction pair potentials. We applied this to decagonal approximants of $\mathrm{Al}_{72} \mathrm{Co}_{8} \mathrm{Ni}_{20}$ and $\mathrm{Al}_{73} \mathrm{Co}_{27}$, obtaining the best energies seen to date for d-AlCoNi models (respectively $+20 \mathrm{meV} / \mathrm{atom}$ and $+9 \mathrm{meV} /$ atom above the tie-plane of competing phases). We elucidated the reasons that different decagonal structures are associated with the Ni-rich and Co-rich compositions. We find a cell doubling to $c=8 \AA$ due to layer puckering not only in the Co-rich structure, but (locally) in the nominally $c=4 \mathrm{~A}$ Ni-rich structure.
\end{abstract}

Keywords: molecular dynamics; effective Hamiltonian; Al-TM; ab-initio energies

\section{1. introduction}

This work is part of an effort to understand the structure and stability of decagonal $d$-AlNiCo, the highest quality decagonal quasicrystal. Due to the complexity of the structure, and averaging-over-disorder effects, even good diffraction datasets $[1,2]$ cannot reliably provide the precise structure - not well enough to predict the well-known fact that $d$-AlCoNi is thermodynamically stable with respect to the competing crystal phases owing to their excess energy relative to competing crystals.

Previous simulation studies derived low-energy structural models on the $\mathrm{Ni}$ rich $[3,4]$ and Co-rich [5-7] compositions, or on the whole range [8] by first Monte Carlo annealing a lattice-gas on discrete sites. (augmented by tile-reshuffling moves). The prerequisites for that method were (i) realistic pair potentials; (ii) "minimal experimental input" in the form of the layer spacing and quasilattice constant (i.e tile size); (iii) most importantly the fact of layering, and the assumption of near-ideal candidate atomic positions.

In agreement with experiment, these studies arrived at similar but distinct structure types in the Ni-rich and Co-rich cases, which we will call "Ni type" and "Co type" for short. The Ni-type structure is based on hexagon/boat/star HBS) tiles (at a hierarchy of length scales [3]) and, according to experiment, has a stacking

*Corresponding author. Email:mihalkovic@savba.sk 
period of 4 Aalong the 10-fold axis; the HBS tiles group into (possibly overlapping) decagonal clusters with diameter $20 \AA$, having central motifs with (only) mirror symmetry, appearing as triangles of TM atoms in high-resolution electron microscope images.

The Co-type structure, although it contains HBS tiles also, is dominated by 20 A non-overlapping decagon clusters which we shall call "pentagonal decagons" (PD). Other prominent features are clusters similar to pentagonal bipyramids (PB), centered on the perimeter of the $20 \mathrm{~A}$ decagons and forming columns in which some $\mathrm{Al}$ atoms pucker out of the layers in alternate directions, causing a cell doubling to $c=8 \AA$ in the stacking direction.

We believe the key reason for the excess energy is the displacement of certain $\mathrm{Al}$ atoms pucker out of the main atom layers, called "puckering". This is explained in terms of "channels": [4-6] linear potential wells for Al atoms that are aligned transverse to the atom layers, located between two columns of TM atoms. The Al atoms, when packed tightly into the channels, must be spaced $\sim 2.6 \mathrm{~A}$, i.e. 1.3 layers apart,so that three Al atoms fit in four layers. Hence locally in any channel, the stacking period gets doubled from two to four layers; only one of the three atoms aligns with a layer, which becomes the local mirror plane of that channel. Puckerings occur, as a form of local order, even in alloys whose average structure is just two layers. Consequently, diffuse scattering [9-12] is observed corresponding to the 4-layer local order, and this diffuse scattering shows elaborate modulations within the 10-fold layer due to correlations between the phases of puckering in nearby "channels". Thus a successful understanding of those correlations will also give the first theory for this diffuse scattering.

Structure models without puckering at all are many tens of meV/atom too high in energy. Allowing puckering locally can capture half of this energy but is still not sufficient to obtain the lowest energies. For that, we must incorporate the correct correlations between the signs of puckering of $\mathrm{Al}$ atoms in nearby channels. since these atoms interact strongly. (A weakness of our past approach $[3,5]$ is that the discrete site list used initially does not incorporate puckering displacements.) No simple recipe is known for the global puckering pattern. Note that $d$-AlNiCo has a well-known zoo of structural modifications [19] which depend on tiny differences in composition, and (we suspect) go with different puckering patterns.

\section{Cell-constrained melt-quench method}

One of the most challenging tasks of contemporary material science, and the basic question in our studies of quasicrystal structures is: given a composition, what is the optimum structure? A general method was put forward using ideas of genetic algorithms and including variation of the cell, as implemented in the program USPEX [13], but apparently this cannot yet handle systems with complex structure.

We have developed an approach we call "cell-constrained melt-quench" which is surprisingly successful at predicting low-temperature structures of complex alloys with hundred(s) of atoms per cell. This simply means cooling the system under brute-force molecular dynamics using periodic B.C.'s in a small cell, while fixing the composition, atomic density, and the unit cell of the system to the experimentally known values. Quite often those are available experimentally from electron diffraction or powder diffraction, when single-crystal structure refinement is impossible. The "cell constraint" seems to limit the ensemble of possible states so we can shepherd the system to the global energy minimum.

One's expectation is that, in a multi-species system described by oscillating in- 


\section{Page 3 of 11 \\ Philosophical Magazine \& Philosophical Magazine Letters}

AlCoNi from DOE08

teratomic potentials [3], such a quench should get caught in a glassy or highly defective metastable state rather than the true energy minimum. But we found it was sufficient to apply the trick of "replica exchange" ("parallel tempering") [14], wherein a set of simulations is run, each at a different temperature, and the entire configuration may be exchanged between temperatures according to a Metropolis step. (Note that within each loop, we perform not only MD but also MC steps that swap the species of two atoms.)

Of course, it is also crucial to have realistic pair potentials. These may be constructed systematically (as with Moriarty's GPT potentials [26] which we used in this project). Alternatively, for Al-transition metal (Al-TM) and many other alloys, one does just as well with empirical potentials including Friedel oscillations that can quickly be fitted to an appropriate database of ab-initio energies and forces. [16] Empirical potentials (specified by a few parameters) may be fitted to data sets of ab-initio forces and energies. Alternatively, analytical potentials are available for certain systems, in particular GPT potentials [26] were developed for Al-rich Al-TM systems.

The general philosophy of this method - taking advantage of lattice and composition information to moderately constrain the simulation - also inspired our earlier work based on quasi lattice-gases $[3,7]$. In the present approach, we need fewer assumptions, and the method directly finds good answers for the (intricate) patterns of atoms deviating out of the layers, which turns out to be the crucial unresolved aspect of the structure model.

The cell-constrained method has been successful for a variety of intermetallics [17]. For example, in the $i$-CaCd family of structures, this method successfully gave the starting point for a Rietveld refinement of the " $2 / 1-1 / 0-1 / 0$ " $\mathrm{AlCuSc}$ approximant [15].

\section{Set up for Al-Co-Ni melt-quench study}

Our simulations used the realistic GPT pair potentials [26] as we used before [3, 5, 7]. The cell sizes for decagonal approximants are all based on tilings with lattice constants $a_{q}=2.44 \AA^{\circ}$ in-plane, and $c=4.08 \AA^{\circ}$ for the minimum stacking period.

The melt-quench (MQ) simulation was set up with 20 annealing temperatures distributed between $1000 \mathrm{~K}-2500 \mathrm{~K}$. One tempering cycle consisted of $1000 \mathrm{MD}$ steps with time increment $\Delta T=0.2 \mathrm{fs}$, followed by a Metropolis Monte Carlo (MC) loop of 7800 attempted species swaps (the acceptance rate was 0.03-0.05). Typically, for a system of this size the simulation needs $~ 100$ steps until first metastable lowenergy configurations appear. Occasionally, we take the current lowest-temperature sample and quench it to monitor the energy of the underlying zero-temperature state.

To validate our method, we first verified that cell-constrained quenching with GPT potentials [26] reproduced all Al-rich binary structures in the Al-Co and $\mathrm{Al}-\mathrm{Ni}$ systems, as well as the ternary phase $X$-AlCoNi.

With $\mathrm{Al}-\mathrm{Ni}$, we verified that $12 \mathrm{Al}+4 \mathrm{Ni}$ in the orthorhombic unit cell of $\mathrm{Al}_{3} \mathrm{Ni}$ $\left(16\right.$ atoms $\left.6.6 \times 7.4 \times 4.8 \mathrm{~A}^{\circ}\right)$ easily reproduced that structure after just 10 loops. On the Co-rich side, the essential test was the orthorhombic $\mathrm{Al}_{13} \mathrm{Co}_{4}$ phase; the monoclinic (fully occupied) version $m-\mathrm{Al}_{13} \mathrm{Co}_{4}$ with 51 atoms per primitive cell was exactly recovered after 119 loops. We also reproduced $\mathrm{Al}_{9} \mathrm{Co}_{2}$.

Finally, we checked the ternary $\mathrm{X}(\mathrm{AlCoNi})$ phase [23] which has ratio 18:5:3 Al:Co:Ni in a monoclinic unit cell: the structure was observed after 71 loops. The longest of the test simulations $\left(\mathrm{m}-\mathrm{Al}_{13} \mathrm{Co}_{4}\right)$ needed just a few minutes on a standard 
Figure 1. Various outcomes of constrained-cell melt-quench simulation, (a). The " $2 \mathrm{~B}+\mathrm{H}$ " unit cell is tractable on the Co-rich side, here $\mathrm{Al}_{158} \mathrm{Co}_{52}$. Here and in Fig. 2, the view is along the short (c) axis; atoms are marked by circles, with larger sizes denoting larger $z$ coordinate. Lines show bonds between atoms in different layers heavy and light for TM-TM and Al-Al, with projected lengths $4.1 \pm 0.3 \mathrm{~A}^{\circ}$ and $2.6 \mathrm{~A}^{\circ}$, respectively. (b). A B2-like structure found in the same cell for Ni-rich composition $\mathrm{Al}_{148} \mathrm{Co}_{16} \mathrm{Ni}_{41}$ viewed along the $(1,1,0)$ direction) ( $c$ axis is horizontal). (c). Co-rich "Co-type" MQ structure $\mathrm{Al}_{58} \mathrm{Co}_{14} \mathrm{Ni}_{9}$, "Boat" cell. Composition similar to W-phase [24].

quad-core PC, demonstrating the efficiency of the method for system sizes up to $\sim 50$ atoms per simulation box.

First we show the results of simulations in a larger unit cell we call the " $2 \mathrm{~B}+\mathrm{H}$ cell" since it accommodates two Boat tiles and one Hexagon tile of edge $6.5 \AA$ per primitive cell; the cell is centered orthorhombic with cell parameters (in a monoclinic setting) $a=b=19.78 \AA^{\circ}, \gamma=108^{\circ}$, and $c=8.06 \AA$. With composition $\mathrm{Al}_{0.75} \mathrm{Co}_{0.25}$, we obtain a decagonal approximant [Fig. 1(a)] On the other hand, using our standard Ni-rich composition $\mathrm{Al}_{148} \mathrm{Co}_{16} \mathrm{Ni}_{41}$ in this cell, we obtain the B2 structure [Fig.1(b)].

The chief technical hindrance to obtaining decagonal approximants is competition from the B2 phase (Fig. 1(b), which has ideal composition $\mathrm{Al}(\mathrm{Co}, \mathrm{Ni})$ in which Co and Ni substitute each other. Since B2 has a small unit cell, it fits with moderate distortion into almost every one of our simulation cells. B2 is a thermodynamically stable phase at high temperatures, and is probably more accessible than the desired phases. We ascribe both properties to the very large configurational entropy of $\mathrm{Al}$ atoms substituting on TM sites (as necessitated by our Al-rich compositions.)

Due to their unrealistically deep Al-TM nearest-neighbor wells, the GPT pair potentials exaggerate the stability of B2. Still, it is known experimentally that B2$\mathrm{Al}(\mathrm{Co}, \mathrm{Ni})$ (with $\mathrm{TM}$ site disorder) does coexist with decagonal phases in $\mathrm{AlCoNi}$ samples carefully annealed at high $T$ [18]. 


\section{Page 5 of 11 \\ Philosophical Magazine \& Philosophical Magazine Letters}

Figure 2. (a,b) Ni-type or Co-type structures emerge in the "Boat" cell, for (respectively) lower density $\left(\mathrm{Al}_{56} \mathrm{Co}_{6} \mathrm{Ni}_{16}\right)$ or higher density $\left(\mathrm{Al}_{58} \mathrm{Co}_{6} \mathrm{Ni}_{16}\right)$. (c). Electronic density of states e(DOS) for three cases: Co-type Co-rich $\left(\mathrm{Al}_{58} \mathrm{Co}_{14} \mathrm{Ni}_{9}\right.$ in Fig. 1c), Co-type Ni-rich (Fig. 2b) and Ni-type Ni-rich (Fig. 2a).

\section{Results}

To avoid formation of the spurious B2 phase, we adopted a simulation cell smaller in linear dimensions by a factor $\tau \equiv(1+\sqrt{5}) / 2 \approx 1.618$ that we believe matches the $\mathrm{B} 2$ phase poorly. It is centered orthorhombic with lattice constant $a=b=$ $12.3 \mathrm{~A}^{\circ} \gamma=108^{\circ}$ in monoclinic setting, and $c=8.20 \AA^{\circ}$ (so the layer spacing is $2.05 \AA$ ). Each primitive cell accommodates one $\tau^{2}$-inflated Boat tile of edge $6.4 \AA$. [In orthorhombic setting the cell is $a=14.36 \AA^{\circ}, b=19.69 \AA^{\circ}$ and $c=8.16 \AA$..] Also, the approximate composition of the Ni-rich decagonal is reported to be $\mathrm{Al}_{72} \mathrm{Co}_{8} \mathrm{Ni}_{20}$. So we studied various cell contents, most importantly $\mathrm{Al}_{56} \mathrm{Co}_{6} \mathrm{Ni}_{16}$ at the Ni-rich end (with variations having $57 \mathrm{Al}$ and/or $15 \mathrm{Ni}$ ), and $\mathrm{Al}_{58} \mathrm{Co}_{14} \mathrm{Ni}_{9}$ at the Co-rich end.

Atomic density is a key parameter; experimentally reported values in $d$-AlCoNi vary from 0.066 atoms $/ \mathrm{A}^{3}$ according to [22] up to 0.070 atoms $/ \mathrm{A}^{3}$ (see References in [21]). Our runs explored this whole range which corresponds to 77-80 atoms per primitive cell. We call a structure "high density" when it has 58 or $57 \mathrm{Al}$ atoms in the "Boat" cell (see Fig. 2)

As expected, we could obtain both Ni-type and Co-type decagonal structures in different compositions. What was unclear a priori was (i) are these two discrete possibilities, or is there a gradual continuum of types interpolating between them? (ii) what determines which of the types is seen? 


\section{Puckering}

We find not only Co-rich but Ni-rich structures are locally $8 \mathrm{~A}$ periodic. A convenient summary of the puckering behavior is seen in the distribution function of the atoms' $z$ coordinates, as shown by insets in each figure.

The Co-type structures all contain four $2 \mathrm{~A}$ layers: two perfectly flat (mirror) layers alternating with two puckered layers. The reasons for this kind of ordering were laid out in Ref. [6]; a long range order can propagate because there is a well connected network of channels across the system. The $\mathrm{Al}_{58} \mathrm{Ni}_{22}$ sample (in a Co-type structure) shows the same puckering behavior as the Co-rich sample.

By contrast, in the Ni-type structures found at lower atomic densities all four layers have some puckered atoms: Fig. 2(a), inset). That means each layer has a mixed nature: for some "channels" it is a (local) mirror layer, but for others it is a (local) puckered layer. Presumably the Ni-type system has statistical $4 \mathrm{~A}^{\circ}$ period despite its local $8 \AA^{\circ}$ ordering, because the puckered motifs are rarer and less correlated.

In both types of decagonal structures, one finds certain columns of TM atoms surrounded by five neighboring TM columns $4 \mathrm{~A}^{\circ}$ away. In the Ni-type structure this happens at the center of every Star tile of the small HBS tiling in Fig. 2(a). In the Co-rich type structure [6], it occurs on every other vertex of the D-ring which bounds the $12 \mathrm{~A}$ Decagon (Fig. 2(b). Such columns are surrounded by five "channels", which have strongly correlated puckering patterns due to steric constraints. In Ref. [6] we identified a characteristic motif of such "puckering centers", called the "crooked cross", and it is in fact visible in every puckering center in Figs. 1(a) and $2(\mathrm{a}, \mathrm{b})$. This consists (in projection) of a pair of puckered Al atoms at the same $z$ and on opposite sides of the central TM; a second such pair lies at a different $z$ and (in projection) roughly at right angles to the first. (These atoms stand out in the figures because they are the only ones whose projections do not lie on ideal quasilattice sites.)

Finally, the $\mathrm{Al}_{0.75} \mathrm{Co} .25$ structure of Fig. 1(a) has the same puckering pattern as 


\section{Page 7 of 11 \\ Philosophical Magazine \& Philosophical Magazine Letters}

AlCoNi from DOE08

in the well-known $\mathrm{Al}_{13} \mathrm{Co}_{4}$ structure. Some puckered atoms are an intrinsic part of the "pentagonal bipyramid" motif [20,21]; on top of the same cluster there is an atom pair, as in a "crooked cross", but the other arm of the cross is missing, because an $\mathrm{Al}$ atom appears right on the local 5 -fold axis at the same $z$ (a mirror-layer) that the second two atoms would have been (and excludes them sterically).

To demonstrate how essential the local puckering is, we ran the same Ni-rich composition while replacing the $8 \mathrm{~A}^{\circ}$ cell constraint by $4 \mathrm{~A}^{\circ}$. The resulting structure contains no clearly pentagonal motifs; instead, it somewhat resembles the $4 \mathrm{~A}$ periodic phase $\mathrm{X}(\mathrm{AlCoNi})$ [23]. Although its pair-potential energy is practically degenerate with the $(8 \AA)$ decagonal's, we never observed the $4 \mathrm{~A}^{\circ}$ phase in the $8 \AA$ box: presumably its entropy is too small.

\section{Ab-initio calculations of phase stability}

Having obtained structures from cell-constrained simulations with pair potentials, we check their (relaxed) energy a posteriori using the ab initio program VASP [27]. Our criterion for stability is a zero-temperature energy of at most $10 \mathrm{meV} /$ atom above the tie-plane of other phases. Note that a small excess energy is often compensated in quasicrystals and approximants by a higher vibrational entropy not found in other alloy structures, meaning the quasicrystal, or approximant, is stable as a high-temperature phase.

For the Al-Co alloy system [21] and the Al-Ni binary and also the Al-Co-Ni ternary system, we evaluated the total energies of all experimentally stable Alrich phases (except $\epsilon-\mathrm{Al}_{3} \mathrm{Co}$, which at 342 atoms/cell is too large to handle). We constructed the convex hull of coexistence tie-planes on the plot of energy as a function of composition; our energies are quoted relative to the convex hull defined by $\mathrm{Al}_{3} \mathrm{Ni}, \mathrm{Al}_{3} \mathrm{Ni}_{2}, \mathrm{Al}_{9} \mathrm{Co}_{2}$, and $\mathrm{Al}_{5} \mathrm{Co}_{2}$.

We pause for a moment to sketch the "energy landscape" of these competing phases. $X-\mathrm{Al}_{9} \mathrm{Co}_{2} \mathrm{Ni}_{2}$ had energy $8.5 \mathrm{~A}^{\circ}$ above the tie-line $[23](+6.1 \mathrm{meV}$ for composition $\mathrm{Al}_{9} \mathrm{Co}_{2.5} \mathrm{Ni}_{1.5}$ ), and $W$-AlCoNi had energy $+12.0 \mathrm{meV}$ [25]. $\mathrm{Al}_{9} \mathrm{Co}_{2}$ remains stable in a ternary composition (replacing $\mathrm{Co} \rightarrow \mathrm{Ni}$ ) up to $9 \% \mathrm{Ni}$. Note that [21], $\mathrm{Al}_{13} \mathrm{Co}_{4}$ is a high-temperature phase, stabilized by vibrational and vacancy/substitutional entropy; at full occupancy, it is unstable by $10 \mathrm{meV} /$ atom.

The decagonal approximant structures we obtained had the best energies seen to date for any d-AlCoNi models with the Ni-rich and Co-rich composition respectively $+20 \mathrm{meV} /$ atom (Fig.2(a)) and $+19 \mathrm{meV} /$ atom (Fig.1(c)) above the convex hull of low-temperature competing phases. It turns out that $\mathrm{Al}-\mathrm{Co}$ has quite good energies in typical $\mathrm{Al}-\mathrm{Ni}$ structures (e.g. nearly stable in the $\mathrm{Al}_{3} \mathrm{Ni}$ structure), but not vice versa: structures favorable for $\mathrm{Al}-\mathrm{Co}$ e.g. $\left(\mathrm{Al}_{13} \mathrm{Co}_{4}\right.$ or $\left.\mathrm{Al}_{5} \mathrm{Co}_{2}\right)$ are highly unfavorable in the $\mathrm{Al}-\mathrm{Ni}$ system. The well ordered Co-type structures are unstable with Ni-rich compositions, by $+53 \mathrm{meV} /$ atom $\left(\mathrm{Al}_{58} \mathrm{Co}_{6} \mathrm{Ni}_{16}\right)$. For lower densities, optimal structures were always Ni-type: unstable by $+36 \mathrm{meV} /$ atom $\left(\mathrm{Al}_{57} \mathrm{Co}_{6} \mathrm{Ni}_{16}\right)$, optimally $+20 \mathrm{meV} /$ atom $\left(\mathrm{Al}_{56} \mathrm{Co}_{6} \mathrm{Ni}_{16}\right)$, and by $+24 \mathrm{meV} /$ atom at even lower density cell content $\left(\mathrm{Al}_{56} \mathrm{Co}_{6} \mathrm{Ni}_{15}\right)$. For Al-Co the best decagonallike structure is always Co-type; in particular, $\mathrm{Al}_{0.75} \mathrm{Co}_{0.25}$ in Fig. 1(a) is just 7.5 $\mathrm{meV}$ /atom unstable against decomposition to $\mathrm{Al}_{9} \mathrm{Co}_{2}$ and $\mathrm{Al}_{3} \mathrm{Co}$.

We now come to the point, why (in reality) the (nearly) stable decagonal-type structures are Ni-type for Ni-rich compositions and Co-type for Co-rich compositions. For Co fraction above $\sim 25 \%$ the $\mathrm{Al}-\mathrm{Co}$ binary is destabilized by competition with $\mathrm{Al}_{5} \mathrm{Co}_{2}$ : nothing breaks structurally in the decagonal, but the tie-line is simply pulled down by the very good $\mathrm{Al}_{5} \mathrm{Co}_{2}$ energy. Conversely, in the $\mathrm{Al}-\mathrm{Ni}$ system, 


\section{Discussion}

In summary, we obtained excellent structural energies from direct quenching molecular dynamics with pair potentials, when constrained by cell parameters and aided by realistic potentials as well as replica-exchange and other Monte Carlo steps. The size limit on our unit cell was not from the computer time needed to anneal it, but rather from the need to ensure that unwanted high-temperature phases are mismatched with the lattice parameters. We found there are two discrete structure types, and explained (Sec. 6) why one goes with Ni-rich compositions and the other with Co-rich compositions. Ultimately we believe the physical parameter controlling the phase is electron density (which tracks atom density): higher or lower electron densities are optimal for Co-rich or Ni-rich compositions, respectively.

There is no hope to realistically model any decagonal without (at least local) $8 \AA$ period due to "puckering" of $\mathrm{Al}$ atoms out of their layers, as emphasized by the failure to obtain a decagonal in a $4 \mathrm{~A}^{\circ}$ cell. Note that the best energy for Nirich $d$-AlCoNi found here is comparable to the best energy found by hand-built variations and/or MD-relaxations applied to tile-decoration models [29] approach. Thus, each structure includes some feature that the other one lacks. The cellconstrained quench finds the large-scale feature of the D-ring, as well as local puckerings, but probably does not find the absolute best global correlations of the puckerings. The decoration variations are based on a hierarchical pattern of tilings [3] with edges $\sim 2.5 \AA^{\circ}, \sim 4.0 \AA^{\circ}$, and $\sim 6.5 \AA^{\circ}$, which may be superior to the quench results (which have well defined TM positions but some Al variability); furthermore, due to a more systematic testing of puckering patterns, they may have achieved better puckering correlations.

The cell-constrained quench, thus, may ultimately not be a direct shortcut to good structures. Rather, it might replace lattice-gas Monte Carlo as a better method to use in the early stages of our recipe $[3,5]$, where one is discovering basic decoration rules which permit further simulations at larger length scales. It will be interesting to see if decorations which ensure a high density of D-rings can reach energies as low as the melt-quench structures.

\section{Acknowledgments}

Supported by U.S. Dept of Energy grant DE-FG02-89ER-45405 (CLH) and by Slovak VEGA 2/0157/08. CLH acknowledges a travel fellowship from the Slovak Academic Information Agency (2010). 


\section{Page 9 of 11 \\ Philosophical Magazine \& Philosophical Magazine Letters}

REFERENCES

\section{References}

[1] A. Cervellino, T. Haibach, and W. Steurer, Acta Crystallogr. B 58 (2002) pp. 8-33.

[2] H. Takakura, A. Yamamoto, and A. P. Tsai, Acta Crystallogr. A 57 (2001) pp. 576-585.

[3] M. Mihalkovič, I. Al-Lehyani, E. Cockayne, C. L. Henley, N. Moghadam, J. A. Moriarty, Y. Wang, and M. Widom, Phys. Rev. B 65 (2002) 104205

[4] C. L. Henley, M. Mihalkovič, and and M. Widom, J. Alloys. Compounds 342, 221-7 (2002).

[5] N. Gu, M. Mihalkovič, and C. L.Henley, Phil. Mag. Lett. 87, 923 (2007).

[6] N. Gu, M. Mihalkovič, and C. L.Henley, unpublished (arxiv.org/abs/cond-mat/0602095).

[7] N. Gu, C. L.Henley, and M. Mihalkovič, Phil. Mag. 86 (2006) p. 593

[8] S. Hiramatsu and Y. Ishii, J. Phys. Soc. Jpn. 75 (2006) art. 054602.

[9] F. Frey and W. Steurer, J. Non-cryst. solids 153 (1993), p. 600.

[10] F. Frey, E. Weidner, K. Hradil, M. De Boissieu, R. Currat, K. Shibata, A. P. Tsai, and T. J. Sato, Phil. Mag. A. 80, 2375-91 (2000)

[11] "Disorder in decagonal quasicrystals", F. Frey and E. Weidner, Z. Kristallogr. 218, 160-169 (2003).

[12] "Structural disorder in the decagonal Al-Co-Ni. I. Patterson analysis of diffuse x-ray scattering data", M. Kobas, T. Weber, and W. Steurer, Phys. Rev. B 71, 224205 (2005).

[13] A. R. Oganov and C. R. Glass: J. Chem. Phys. 124 (200x) art. 244704

[14] R. H. Swendsen and J. S. Wang, Phys. Rev. Lett. (1986), p. 2607; M. E. J. Newman and G. T. Barkema Monte Carlo Methods in Statistical Physics, Oxford, 1999; E. Lyman, F. M. Ytreberg, and D. M. Zuckerman, Phys. Rev. Lett. 96, 028105 (2005).

[15] T. Ishimasa, A. Hirao, T. Honma and M. Mihalkovič, proceedings of ICQ11.

[16] M. Mihalkovič et al, unpublished "Empirical oscillating potentials for alloys from ab-initio fits" (arxiv.org/abs/0802.2926).

[17] M. Mihalkovič and C. L. Henley, unpublished.

[18] B. Grushko, D. Holland-Moritz, R. Wittmann, G. Wilde, J. Alloys Comp. 280 (1998), p. 215.

[19] S. Ritsch, C. Beeli, H. U. Nissen, et al. Philos. Mag. Lett. 78 (1998) pp. 67-75.

[20] C. L. Henley, J. Non-Cryst. Solids 153\&154 (1993), pp. 172-176.

[21] M. Mihalkovic and M. Widom, Phys. Rev. B 75 (2007), art. 014207.

[22] P. J. Steinhardt H. C. Jeong, K. Saitoh, M. Tanaka, E. Abe, and A. P. Tsai, Nature 396 (1998) pp 55-57.

[23] S. Katrych, M. Mihalkovič, V. Gramlich, M. Widom, and W. Steurer, Philos. Mag. 86 (2006) pp. 451-456.

[24] K. Sugiyama, S. Nishimura and K. Hiraga, J. Alloys and Compounds 342 (2002) p. 65.

[25] M. Mihalkovič and M. Widom, Phil. Mag. 86 (2006) p. 557

[26] J. A. Moriarty, Phys. Rev. B c 1996; M. Widom, I. Al-Lehyani and J.A. Moriarty, Phys. Rev. B 62 (2000), p. 3648.

[27] G. Kresse and J. Hafner, Phys. Rev. B 47 (1993), p. R558; G. Kresse and J. Fürthmüller, Phys. Rev. B 54 (1996), p. 11169; G. Kresse and D. Joubert, Phys. Rev. B 59 (1999), p. 1758.

[28] S. Lim, M. Mihalkovič, and C. L. Henley, Philos. Mag. 88 (2008), pp. 1977-1984.

[29] M. Mihalkovič and M. Widom, unpublished. 


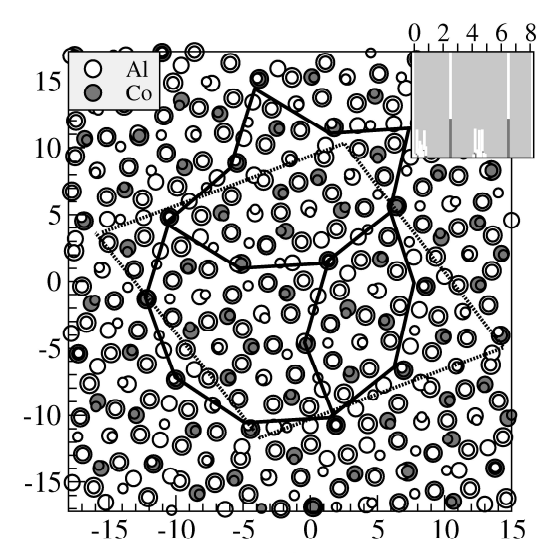

(a)

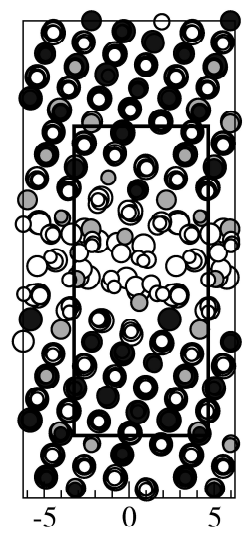

(b)

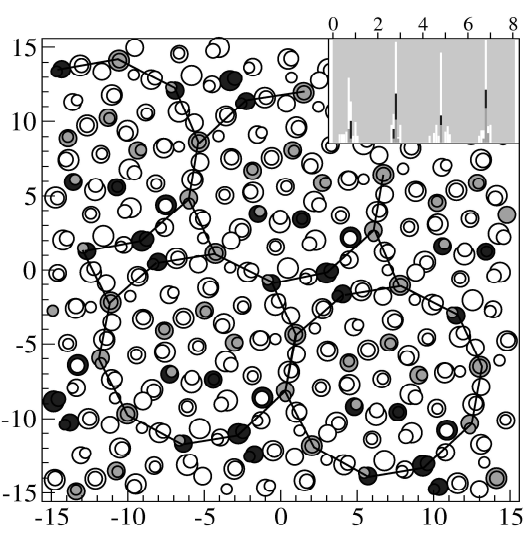

(c)

$262 \times 117 \mathrm{~mm}(600 \times 600$ DPI $)$ 


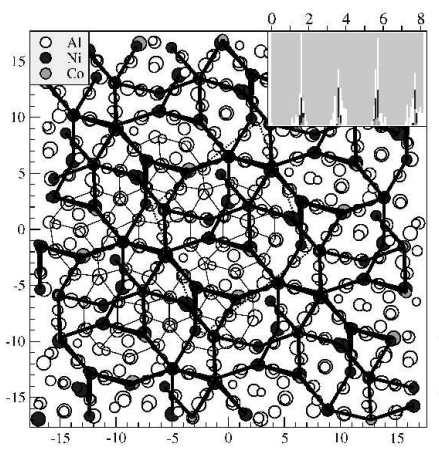

(a)

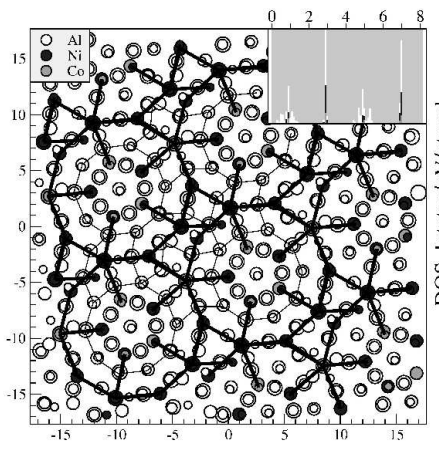

(b)

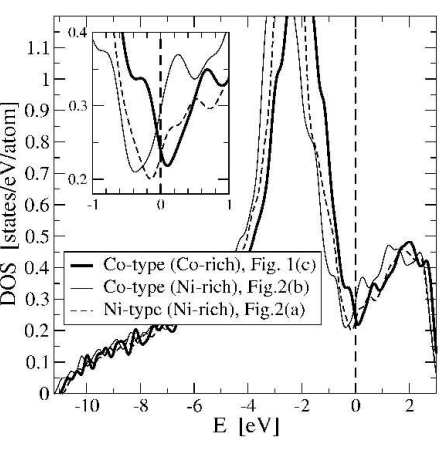

(c)

$285 \times 108 \mathrm{~mm}(600 \times 600 \mathrm{DPI})$ 